\title{
A case of neuroleptic malignant syndrome with aripiprazole and fluoxetine
}

\author{
Tina $\mathrm{Hu}, \mathrm{MD}, \mathrm{MSc}^{*}$; Elise Hall, $\mathrm{MD}^{\dagger}$

\section{CASE REPORT}

A 24-year-old male with a history of schizophrenia presented to the emergency room in an agitated and confused state, with an acute onset of generalized muscular rigidity. He had recently been switched from olanzapine ( $20 \mathrm{mg}$ orally/day) to long-acting aripiprazole ( $400 \mathrm{mg}$ intramuscularly/month), with his second dose 3 weeks prior to the current admission, and was also taking fluoxetine ( $40 \mathrm{mg}$ orally/day) for depression. On examination, he appeared diaphoretic and had an elevated blood pressure, heart rate, and respiratory rate. He was afebrile and tone was increased in the upper and lower limbs. Investigations showed leukocytosis (white blood cell count: $14.7 \times 10^{9} / \mathrm{L}$ ), elevated creatinine $(121 \mu \mathrm{mol} / \mathrm{L})$, and elevated creatine kinase (initially 4,385 IU/L rising to 14,699 IU/L in 4 hours and 23,190 IU/L after another 6 hours). Other investigations were normal (i.e., electrolytes, thyroid stimulating hormone, toxicology screen, urinalysis, computed tomography scan of the brain, blood cultures, and troponins). His medications were held, and he was transferred to the intensive care unit under the suspicion of neuroleptic malignant syndrome (NMS). Intravenous normal saline boluses, intravenous dantrolene $(2.5 \mathrm{mg} / \mathrm{kg})$, and lorazepam to manage agitation were administered. Creatine kinase levels gradually decreased and muscle rigidity improved. The patient was eventually transferred to the psychiatry inpatient team to manage the initiation of a new antipsychotic agent.

\section{DISCUSSION}

NMS is a life-threatening condition associated with antipsychotic use. It has a characteristic tetrad of altered mental status, muscle rigidity, hyperthermia, and autonomic instability (typically developing in that sequence). ${ }^{1,2} \mathrm{NMS}$ is associated with a high mortality rate $(10 \%-20 \%)$ due to the associated autonomic instability and systemic complications and requires early diagnosis and aggressive treatment. ${ }^{2}$

The pathogenesis of NMS is unknown, but it is believed to be related to dopamine receptor blockade. Central receptor blockade in the hypothalamus may lead to hyperthermia and autonomic instability, whereas blockade in the nigrostriatal pathways may lead to parkinsonian-type symptoms such as rigidity. ${ }^{3} \mathrm{NMS}$ risk is highest with the use of typical, high-potency antipsychotics such as haloperidol; however, reports have indicated that all types of antipsychotics (low potency and atypical) and antiemetic agents with dopaminergic blockade (domperidone and metoclopramide) have been associated with NMS. ${ }^{4}$ The time frame between antipsychotic use and onset of NMS is idiosyncratic. ${ }^{3}$ Risk factors for NMS include prior history of NMS, high antipsychotic doses, parenteral administration, recent or rapid increases in dose, switching antipsychotics, dehydration, and concomitant use with lithium, anticholinergic medications, and antidepressants. ${ }^{2}$

Recent reviews have shown that aripiprazole, as used by our patient, leads to an "atypical" NMS phenotype compared with other atypical antipsychotics. Altered mental status and rigidity are universally present, and there is the possibility of more nausea and vomiting, lower peaks of creatine kinase, less hyperthermia and autonomic instability, and less severe and shorter NMS episodes. ${ }^{1}$ The differences in clinical presentation and laboratory findings may be due to aripiprazole's mechanism of action. Instead of strong $\mathrm{D}_{2}$ blockade,

From the *Department of Family and Community Medicine, University of Toronto, Toronto, ON; and tDepartment of Psychiatry, St. Michael's Hospital, Toronto, ON.

Correspondence to: Dr. Tina Hu, Department of Family and Community Medicine, University of Toronto, 61 Queen Street East, 3rd Floor, Toronto, ON M5C 2T2; Email: tina.hu@mail.utoronto.ca 
aripiprazole is a partial agonist of $\mathrm{D}_{2} / \mathrm{D}_{3} / \mathrm{D}_{4}$ and 5-HT ${ }_{1 \mathrm{~A}}$ receptors. ${ }^{1}$ Antipsychotics with stronger $\mathrm{D}_{2}$ blockade, such as olanzapine and risperidone, have been shown to have a stronger association with NMS and higher severity of NMS episodes. ${ }^{1}$ Interestingly, our patient was concurrently taking fluoxetine, a potent inhibitor of CYP2D6. Aripiprazole is metabolized by CYP3A4/CYP2D6 isoenzymes, and co-administration with fluoxetine may have significantly increased plasma concentrations of aripiprazole. Consistent with the reported NMS phenotype associated with aripiprazole, our patient showed altered mental status and rigidity, signs of autonomic instability (i.e., diaphoresis, high blood pressure, and tachycardia), a lower peak of creatine kinase, no hyperthermia, and had a relatively shorter and less severe NMS episode.

There is no specific diagnostic test for NMS. In patients with possible NMS, a thorough history, examination, and laboratory findings such as elevated creatine kinase (greater than 1000 IU/L) can help confirm the clinical diagnosis. ${ }^{1}$ Table 1 shows the Diagnostic and Statistical Manual of Mental Disorders (DSM-5) diagnostic criteria for NMS; however, clinicians should note that NMS is heterogeneous in onset, presentation, and progression. Other nonspecific laboratory findings include leukocytosis with a left shift, electrolyte abnormalities, elevated liver function tests, elevated creatinine, and myoglobinuria from rhabdomyolysis. ${ }^{1}$ Investigations such as brain imaging and lumbar puncture are used to exclude other causes for altered mental status such as neurological disease and infection. ${ }^{1}$ Serotonin syndrome (associated with the use of serotonergic agents) is the most commonly diagnosed related disorder. However, a few notable differences include that, in serotonin syndrome, rigidity is less severe than in NMS, and there are several characteristics not seen in NMS, including hyperreflexia, shivering, myoclonus, ataxia, nausea/vomiting, and diarrhea.

Treatment involves removing the causative agent and implementing supportive care to prevent complications, such as acute renal failure, acute respiratory failure, arrhythmias, disseminated intravascular coagulation, myocardial infarction, seizures, and sepsis. ${ }^{2}$ Frequent monitoring is needed, and intravenous fluids are required to maintain a euvolemic state. Cooling blankets can be used, and anti-hypertensive agents should be used to lower blood pressure if significantly elevated. Benzodiazepines should be used to control agitation as needed. Specific treatments for NMS are based on case reports, and their efficacy is unsupported with varying recommendations on treatment length. ${ }^{2}$ Dantrolene $(1-2.5 \mathrm{mg} / \mathrm{kg}$ intravenously, up to $10 \mathrm{mg} /$ $\mathrm{kg}$ /day), a direct-acting skeletal muscle relaxant, is often used in adults with NMS and has been reported to decrease rigidity and hyperthermia. ${ }^{3}$ It is associated with a risk of hepatotoxicity when used at high doses. ${ }^{3}$ Bromocriptine $\left(\mathrm{D}_{2}\right.$ agonist; $2.5 \mathrm{mg}$ through nasogastric tube every 6-8 hours, up to $40 \mathrm{mg} /$ day) and amantadine (weak, noncompetitive N-methylD-aspartate receptor antagonist; $100 \mathrm{mg}$ orally to a maximum of $200 \mathrm{mg}$ every 12 hours) can also be used. $^{3}$

NMS often resolves within 2 days to 2 weeks. Risk factors for a prolonged course are depot antipsychotics and structural brain disease. Restarting

Table 1. Diagnostic and Statistical Manual of Mental Disorders (DSM-5) diagnostic criteria for neuroleptic malignant syndrome*

1. Exposure to a dopamine antagonist or dopamine agonist withdrawal within the past 72 hours

2. Hyperthermia ( $>38^{\circ} \mathrm{C}$ on at least two occasions, measured orally)

3. Rigidity

4. Mental status alteration (reduced or fluctuating level of consciousness)

5. Creatine kinase elevation (at least four times the upper limit of normal)

6. Sympathetic nervous system lability (defined as at least two of the following):

- Blood pressure elevation (systolic or diastolic $\geq 25 \%$ above baseline)

- Blood pressure fluctuation ( $\geq 20 \mathrm{~mm} \mathrm{Hg}$ diastolic change or $\geq 25 \mathrm{~mm} \mathrm{Hg}$ systolic change within 24 hours)

- Diaphoresis

- Urinary incontinence

7. Hypermetabolism (defined as heart rate increase $\geq 25 \%$ above baseline and respiratory rate increase $\geq 50 \%$ above baseline)

8. Negative workup for infectious, toxic, metabolic, and neurological causes

Information from DSM-5 5 
antipsychotics carries the risk of a recurrent NMS episode. Risk factors for NMS recurrence include high potency antipsychotics, depot antipsychotics, and concomitant use of lithium. Guidelines for restarting antipsychotics to minimize recurrence risk of NMS include waiting at least 2 weeks prior to restarting antipsychotics, using lower potency agents, starting with low doses and titrating up gradually, avoiding dehydration/concomitant lithium, and monitoring for NMS signs. ${ }^{3}$

Keywords: antipsychotics, neuroleptic malignant syndrome, neuroleptics

Competing interests: None declared.

\section{REFERENCES}

1. Murri MB, Bugliani M, Calcagno P, et al. Secondgeneration antipsychotics and neuroleptic malignant syndrome: systematic review and case report analysis. Drugs $R D$ 2015;15:45-62.

2. Berman BD. Neuroleptic malignant syndrome: a review for neurohospitalists. Neurohospitalist 2011;1:41-7.

3. Chandran GJ, Mikler JR, Keegan DL. Neuroleptic malignant syndrome: case report and discussion. CMAf 2003;169:439-2.

4. Trollor JN, Chen X, Chitty K, Sachdev PS. Comparison of neuroleptic malignant syndrome induced by first-andsecond-generation antipsychotics. $\mathrm{Br} \quad \mathrm{f}$ Psychiatry 2012;201:52-6.

5. American Psychiatric Association. Diagnostic and statistical manual of mental disorders (DSM-5®). Arlington, VA: American Psychiatric Publishing; 2013. 